\title{
The Lagrangian picture of heat transfer in convective turbulence
}

Maik Boltes, Herwig Zilken, and Jörg Schumacher

Citation: Chaos 20, 041109 (2010); doi: 10.1063/1.3497270

View online: https://doi.org/10.1063/1.3497270

View Table of Contents: http://aip.scitation.org/toc/cha/20/4

Published by the American Institute of Physics

\section{Articles you may be interested in}

Nonlinear time-series analysis revisited

Chaos: An Interdisciplinary Journal of Nonlinear Science 25, 097610 (2015); 10.1063/1.4917289

Partial synchronization in diffusively time-delay coupled oscillator networks

Chaos: An Interdisciplinary Journal of Nonlinear Science 22, 043144 (2012); 10.1063/1.4771665

Understanding cardiac alternans: A piecewise linear modeling framework

Chaos: An Interdisciplinary Journal of Nonlinear Science 20, 045102 (2010); 10.1063/1.3518362

\section{Smartersearch $0 \begin{aligned} & \text { with the redesigned } \\ & \text { Physics Today Buyer's Guide }\end{aligned}$}

Find the tools you're looking for today! 


\section{The Lagrangian picture of heat transfer in convective turbulence}

\author{
Maik Boltes, ${ }^{1}$ Herwig Zilken, ${ }^{1}$ and Jörg Schumacher ${ }^{2}$ \\ ${ }^{1}$ Jülich Supercomputing Centre, D-52425 Jülich, Germany \\ ${ }^{2}$ Technische Universität Ilmenau, D-98684 Ilmenau, Germany \\ (Received 9 August 2010; published online 30 December 2010) \\ [doi:10.1063/1.3497270]
}

Convective turbulence can be generated in a fluid layer which is heated from below and cooled from above when the temperature difference between the top and the bottom layer is sufficiently large. Convective turbulence is present in the interior of stars and planets and in planetary atmospheres. Most studies of convective turbulence, particularly those with a focus on the mechanisms of turbulent heat transfer, have been conducted in the Eulerian frame (see Ref. 1).

The Lagrangian perspective on convective turbulence implies that one follows massless tracer particles and monitors velocities, accelerations, and temperature along the tracer tracks. Recently, we have carried out threedimensional direct numerical simulations of the Boussinesq equations of motion. ${ }^{2,3}$ The fluid is enclosed in a rectangular slab of aspect ratio 4 . Periodic boundary conditions are applied in the horizontal directions and free-slip boundary conditions in the vertical direction. The Rayleigh number is $\mathrm{Ra}=1.2 \times 10^{8}$ and the Prandtl number $\operatorname{Pr}=0.7$. A pseudospectral method is used to advance the equations of motion on a grid with $2048 \times 2048 \times 513$ points. In total, we monitored $1.2 \times 10^{6}$ tracer particles.

The upper panel of Fig. 1 shows a snapshot of the Lagrangian tracer ensemble. The particles are colored according to the temperature at their position, which is indicated in the color legend. All tracers were initially seeded in a plane very close to the bottom boundary. White tracers were additionally arranged in a regular grid in order to illustrate turbulent mixing as time progresses. The diagonal cutting plane nicely highlights the coherent thermal plumes. The heat transport in turbulent convection is concentrated in coherent structures that detach from the thin thermal boundary layers at the top and bottom.

In the middle panel, the most intense local heat transfer events are highlighted in red by taking the product of the vertical velocity $u_{z}$ with the temperature fluctuation about the mean temperature profile at the tracer position. Both hot rising plumes from the bottom and cold falling plumes from the top form positive products and thus contribute to the upward heat flux as sketched in the lower panel.

Supercomputations were conducted on 4096 cores of the IBM Blue Gene/P JUGENE at the Jülich Supercomputing Centre. Support by the Deutsche Forschungsgemeinschaft (DFG) is also gratefully acknowledged.
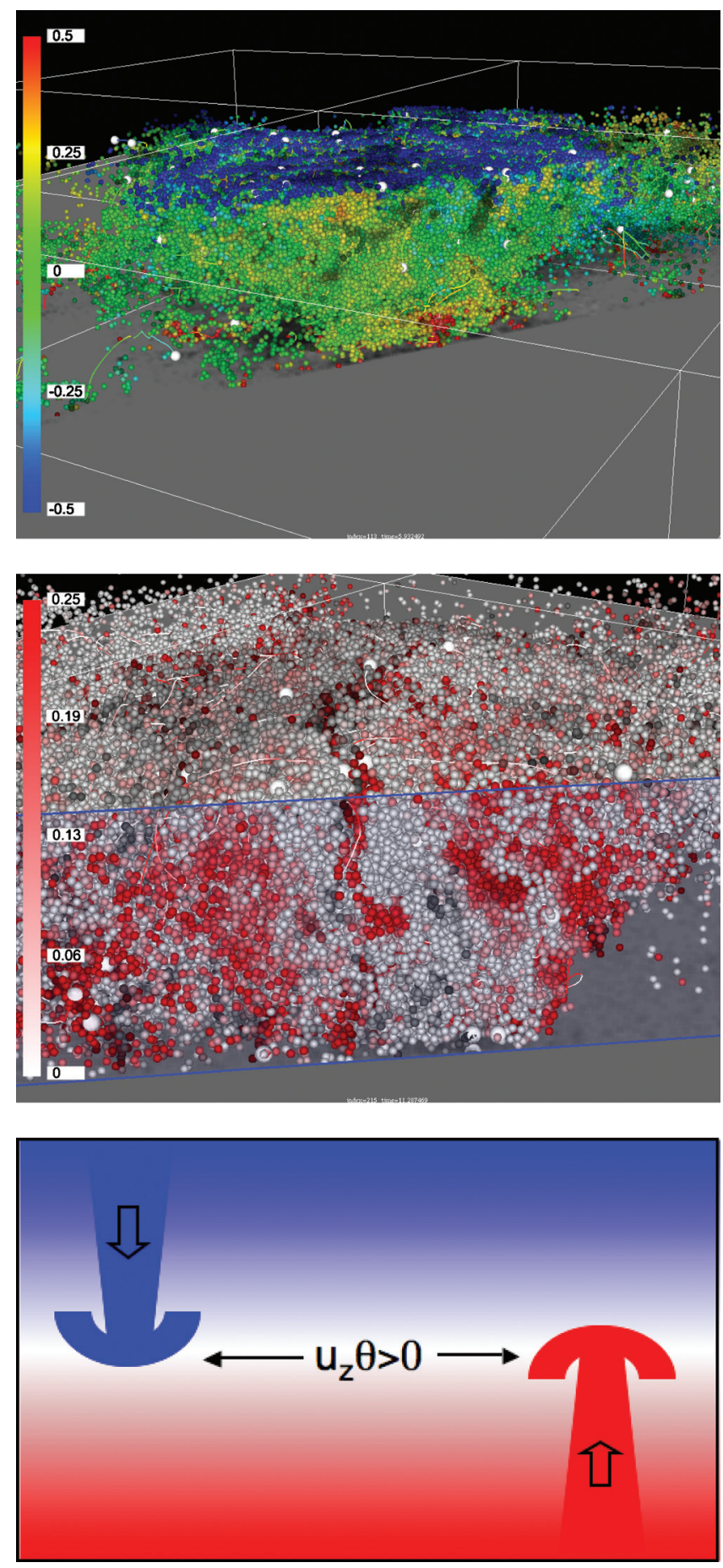

FIG. 1. (Color) (enhanced online).

[URL: http://dx.doi.org/10.1063/1.3497270.1].

${ }^{1}$ G. Ahlers, S. Grossmann, and D. Lohse, Rev. Mod. Phys. 81, 503 (2009).

${ }^{2}$ J. Schumacher, Phys. Rev. Lett. 100, 134502 (2008).

${ }^{3}$ J. Schumacher, Phys. Rev. E 79, 056301 (2009). 